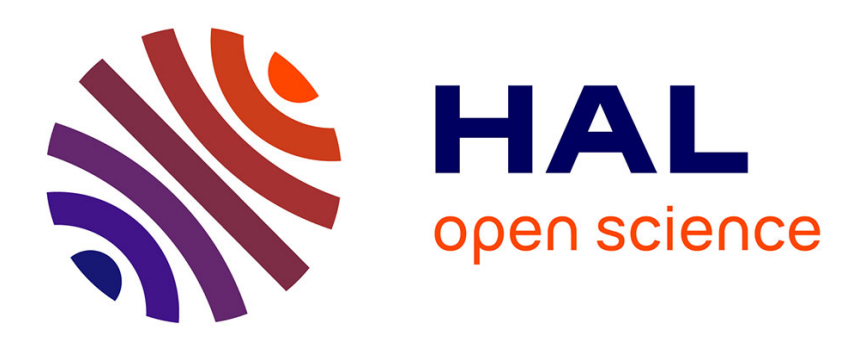

\title{
Noncanonical antibody strategy for broad and potent neutralization of influenza virus
}

Jordan D Dimitrov

\section{To cite this version:}

Jordan D Dimitrov. Noncanonical antibody strategy for broad and potent neutralization of influenza virus. Cellular and molecular immunology, 2021, 10.1038/s41423-020-00617-0 . hal-03146589

\section{HAL Id: hal-03146589 \\ https://hal.sorbonne-universite.fr/hal-03146589}

Submitted on 19 Feb 2021

HAL is a multi-disciplinary open access archive for the deposit and dissemination of scientific research documents, whether they are published or not. The documents may come from teaching and research institutions in France or abroad, or from public or private research centers.
L'archive ouverte pluridisciplinaire HAL, est destinée au dépôt et à la diffusion de documents scientifiques de niveau recherche, publiés ou non, émanant des établissements d'enseignement et de recherche français ou étrangers, des laboratoires publics ou privés. 


\title{
RESEARCH HIGHLIGHT
}

\section{Noncanonical antibody strategy for broad and potent neutralization of influenza virus}

\author{
Jordan D. Dimitrov ${ }^{1}$ \\ Cellular \& Molecular Immunology _\#\#\#\#\#\#\#\#\#\#\#\#\#\#\#\#\#\#; https://doi.org/10.1038/s41423-020-00617-0
}

Pathogens with high levels of genetic variability, such as HIV-1 and influenza virus, evade the immune system by constantly changing their proteins vulnerable to neutralization by antibodies Consequently, these highly evolvable viruses are notoriously elusive targets for vaccines. In rare cases, infection of humans with HIV-1 or influenza virus results in production of antibodies that can neutralize a very broad spectrum of viral strains. Such antibodies are referred to as broadly neutralizing. ${ }^{1}$ The typical modus operandi of such neutralizing antibodies is recognition with high affinity and blocking by steric hinderance of conserved regions of viral proteins that are important for receptor binding or entry into host cells. ${ }^{1,2}$ This neutralization mechanism can be further supported by antibody functions dependent on constant regions, such as antibody-dependent cellular cytotoxicity. ${ }^{3,4}$ Since the rational induction of broadly neutralizing antibody responses may represent the key for the development of efficient vaccines against rapidly evolving viruses, these antibodies have been the subject of numerous thorough investigations. A recent publication in Immunity by Madsen et al described a nonconventional mechanism for potent and broad neutralization of influenza $B$ virus. $^{5}$

The principal targets of influenza virus recognized by neutralizing antibodies are the envelope glycoproteins hemagglutinin ( $\mathrm{HA})$ and neuraminidase (NA). ${ }^{4}$ These proteins are expressed on the viral membrane (supercapsid) and play critical roles in the life cycle of the virus. HA is a lectin that specifically recognizes terminal sialic acid. Many glycoproteins on the host cell membrane display sialic acid, thus serving as receptors for attachment of the virus. The second most abundant envelope protein, NA, is an enzyme with glycolytic activity; it specifically cleaves terminal sialic acid residues. By virtue of its enzymatic activity, NA mediates release of the virus from its initial retention in the mucosa, which is caused by the interaction of HA with secretory sialic acid-reach glycoproteins (mucins). ${ }^{4}$ The catalytic activity of NA also plays a critical role in the detachment of newly formed viral particles from the membrane of host cells. $\mathrm{HA}$ is the immunodominant envelope protein, i.e., most antibodies produced during infection recognize epitopes on it. ${ }^{2,4} \mathrm{HA}$ is divided into two structural and functional regions - the head, which is responsible for recognition of sialic acid, and the stalk, which takes part in fusion of the viral membrane with the membrane of the host cell. ${ }^{2}$ The head region is the most frequent target of antibody responses; however, these antibodies usually exhibit only limited neutralization breadth. It is noteworthy that antibodies that recognize the stalk of $\mathrm{HA}$, despite being less frequent, possess a better neutralization potential. ${ }^{2,4}$ This can be explained by the highly conserved nature of the stalk region of $\mathrm{HA}$, contrary to the that of the head region. Notwithstanding that the focus of most research has been on antibodies specific for $\mathrm{HA}$, it is known that NA can also be targeted by neutralizing and broadly neutralizing antibody responses. ${ }^{6}$

Influenza B viruses account for one-fourth of influenza infections, and contrary to influenza A viruses, they have not yet caused known pandemics. Nevertheless, the pathogenic potential of influenza B virus is not insignificant; it is responsible for approximately half of deaths in children with influenza infection. ${ }^{5}$ In their study, Madsen et al cloned a set of 21 antibodies from plasmablasts isolated from a patient with influenza $B$ infection. ${ }^{5}$ Most of these antibodies (48\%) were specific for HA; however, seven antibodies (33\%) were specific for NA, and a smaller fraction (19\%) recognized other viral antigens. Further characterization of the anti-NA antibodies revealed that all seven molecules had broad reactivity for divergent strains of influenza $B$ virus. A neutralization assay showed that among the seven antibodies, two, designated 1G05 and 2E01, demonstrated an exceptionally broad and potent virus neutralization capacity. These antibodies were able to neutralize different influenza B virus strains that had accumulated genetic diversity in a time period spanning more than 70 years. In the next step of the study, the authors assessed the potential of the antibodies to inhibit the enzymatic activity of NA. This functional assay revealed that both the most potent antibodies and the most broadly neutralizing antibodies were able to inhibit the hydrolysis of a low-molecular-weight reporter substrate of NA. This result strongly suggests that the epitopes recognized by $1 \mathrm{G} 05$ and $2 \mathrm{E} 01$ overlap with the catalytic site of the enzyme. To dissect the molecular mechanism underlying the neutralization of divergent strains of influenza $B$ virus, the authors performed structural analyses of the complexes formed by an NA tetramer with Fab fragments of $1 \mathrm{G} 05$ and 2E01 using cryoelectron microscopy. These analyses provided evidence that both antibodies use their CDR-H3 loops to establish direct noncovalent interactions with residues in the active site of NA. Thus, these broadly neutralizing antibodies can be regarded as specific inhibitors of the catalytic site of the enzyme NA. To achieve catalytic inhibition, both antibodies rely on unusually long CDR-H3 loops, consisting of 21 and 25 amino acid residues in 1G05 and $2 \mathrm{E} 01$, respectively. Notably, in their interaction with the catalytic site of NA, the amino acid residues of the antibodies establish interactions similar to those observed for complexes of NA with sialic acid or oseltamivir, a therapeutic inhibitor of NA. Thus, these antibodies can be regarded as typical enzyme inhibitors exploiting molecular mimicry. Interestingly, the antibody penetrating deeper

${ }^{1}$ Centre de Recherche des Cordeliers, INSERM, Sorbonne Université, Université de Paris, F-75006 Paris, France

Correspondence: Jordan D. Dimitrov (jordan.dimitrov@sorbonne-universite.fr)

Received: 2 December 2020 Accepted: 4 December 2020

Published online: 14 January 2021 


\section{Steric hindrance Narrow neutralization}

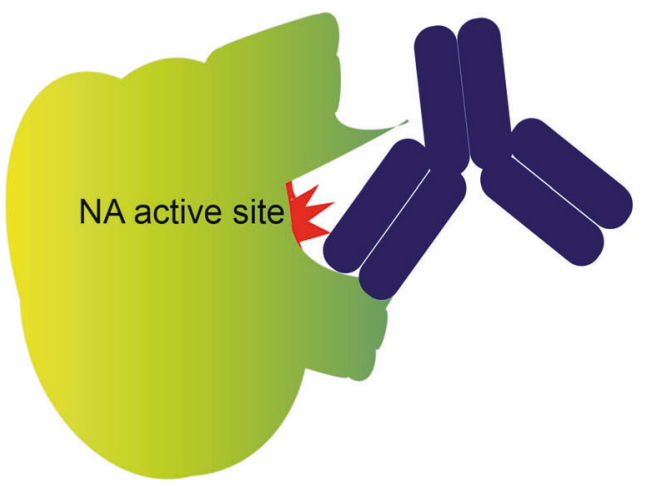

\section{Inhibition of catalytic site Broad neutralization}

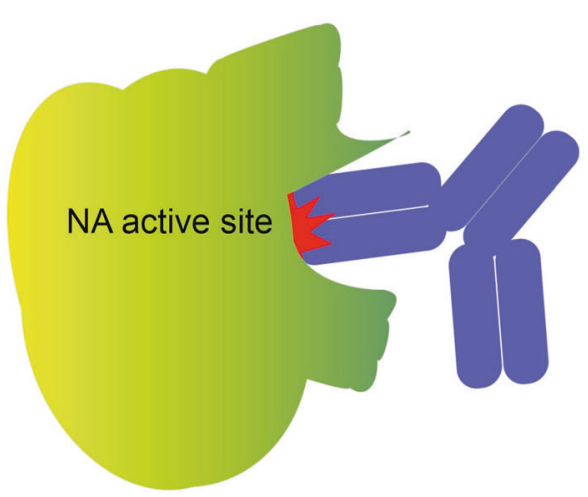

Fig. 1 Modes of neutralization of influenza virus neuraminidase. Neutralizing antibodies can bind in the vicinity of the active site of NA and hamper substrate access by steric hindrance (left). This, however, would result in strain-specific neutralization, as mutations may generate escape variants of NA. The antibodies described in this study interact with the amino acid residues in the catalytic site of NA (right). These antibodies directly block enzymatic activity. Broad neutralization of distinct strains of influenza virus can be achieved, as mutations in the active site are disfavored because they can compromise the functional activity of NA

into the active site of NA (1G05) and establishing more contacts with catalytic residues demonstrated superior inhibition of NA. ${ }^{5}$

Another recent study by the same team reported the detailed characterization of a set of three broadly neutralizing antibodies targeting NA from influenza A viruses. ${ }^{7}$ These antibodies were also able to specifically block NA activity by performing the function of an enzyme inhibitor and establishing a number of direct contacts with catalytic residues in the active site. Collectively, these studies suggest that inhibition of NA catalytic activity may be a common strategy for potent and broad neutralization of both influenza $A$ and $B$ viruses. ${ }^{5,7}$ Both studies present convincing evidence that enzymeinhibiting antibodies have strong protective effects in mouse models of lethal infection with diverse clinically relevant strains of influenza virus. It is noteworthy that enzyme-inhibiting antibodies present not only the capacity to protect animals in the prophylactic setting but also demonstrate considerable therapeutic potential when administered $72 \mathrm{~h}$ after viral challenge in a mouse model.,

Neutralization of viruses by antibodies mostly relays on steric hindrance, which is simply the physical occlusion of the binding site by a bulky molecule, i.e., the antibody. ${ }^{2}$ Virus-neutralizing antibodies also evoke secondary effector systems through interactions with $F C y$ receptors via the Fc portion of the antibody. ${ }^{1,3,4}$ These two strategies work synergistically and can be regarded as conventional mechanisms of neutralization. However, in situations of infection by pathogens with high evolvability, conventional strategies may be unable to control the pathogen. In this case, the adaptive immune response may engage different alternative or unconventional strategies for diversification of variable regions and neutralization of the pathogen. 8 These strategies often occur as a result of the accumulation of a significant number of somatic mutations following multiple iterations of mutagenesis and selection. Importantly, broadly neutralizing antibodies inhibiting the NA enzymatic activity of influenza virus are highly mutated similarly as antibodies using other unconventional strategies for neutralization. ${ }^{5,7}$ The antibodies achieving broad neutralization of a virus with high genetic diversity through interference with catalytic activity can be considered as using another unconventional strategy for pathogen neutralization. This novel neutralization strategy broadens the scope of mechanisms that the humoral immune response can exploit to confront highly variable pathogens, such as influenza virus. The inhibition of functional activity essential for virus physiology in the case of influenza virus represents an efficient tactic for neutralization of diverse strains of this virus. Indeed, enzymatic activity is fundamental for the virus; therefore, extensive variation of the catalytic site to evade antibody neutralization would create a trade off with the loss of enzymatic activity and hence loss of infectivity (Fig. 1).

Antibodies that demonstrate the capacity to block enzymatic activities have already been reported and thoroughly characterized, especially antibodies that inhibit enzymes with protease activity (for example ${ }^{10}$ ). However, these enzyme-inhibiting antibodies have been isolated from synthetic libraries or following immunization. In contrast, the antibodies described here were produced as a result of natural infection of humans.,

The identification of broadly neutralizing antibodies that interfere with the enzymatic activity of NA can have considerable repercussions not only for the basic understanding of the mechanisms of humoral immunity but also for the successful development of vaccines against influenza virus. Thus, immunogens that rationally focus the immune response to recognize the NA active site may contribute to the generation of broadly neutralizing antibodies against influenza virus. Moreover, the monoclonal antibodies that were identified could be used for passive therapy of patients infected with drug-resistant strains of influenza virus. One could also speculate that the sequence of the CDR-H3 loops of broadly neutralizing antibodies could be evaluated to develop peptide inhibitors of NA activity. Collectively, the presented findings represent an important step for successful coping with a sinister virus with prominent pandemic potential.

\section{ACKNOWLEDGEMENTS}

JDD is supported by the Institut National de la Santé et de la Recherche Médicale (INSERM, France).

\section{ADDITIONAL INFORMATION}

Competing interests: The author declares no competing interests.

\section{REFERENCES}

1. Corti, D. \& Lanzavecchia, A. Broadly neutralizing antiviral antibodies. Annu. Rev. Immunol. 31, 705-742 (2013).

2. Murin, C. D., Wilson, I. A. \& Ward, A. B. Antibody responses to viral infections: a structural perspective across three different enveloped viruses. Nat. Microbiol. 4, 734-747 (2019). 
Noncanonical antibody strategy for broad and potent neutralization of... JD Dimitrov

3. DiLillo, D. J., Tan, G. S., Palese, P. \& Ravetch, J. V. Broadly neutralizing hemagglutinin stalk-specific antibodies require $\mathrm{Fc} \gamma \mathrm{R}$ interactions for protection against influenza virus in vivo. Nat. Med. 20, 143-151 (2014).

4. Krammer, K. The human antibody response to influenza $A$ virus infection and vaccination. Nat. Rev. Immunol. 19, 383-397 (2019).

5. Madsen, A. et al. Human antibodies targeting influenza $B$ virus neuraminidase active site are broadly protective. Immunity 53, 852-863 (2020).

6. Krammer, F. et al. NAction! How Can Neuraminidase-Based Immunity Contribute to Better Influenza Virus Vaccines? mBio 9, e02332-e02317 (2018).
7. Stadlbauer, D. et al. Broadly protective human antibodies that target the active site of influenza virus neuraminidase. Science 366, 499-504 (2019).

8. Kanyavuz, A., Marey-Jarossay, A., Lacroix-Desmazes, S. \& Dimitrov, J. D. Breaking the law: unconventional strategies for antibody diversification. Nat. Rev. Immunol. 19, 355-368 (2019).

9. Dimitrov, J. D. \& Lacroix-Desmazes, S. Noncanonical functions of antibodies. Trends Immunol. 41, 379-393 (2020).

10. $\mathrm{Wu}, \mathrm{Y}$. et al. Structural insight into distinct mechanisms of protease inhibition by antibodies. Proc. Natl Acad. Sci. USA 104, 19784-19789 (2007). 